\title{
Effects of Inhaled Nicotine on Instrumental Learning of Blood Pressure Responses ${ }^{1}$
}

\author{
Niels Birbaumer \\ Universitá degli Studii, Padova, Italy and Universität Tübingen, Germany \\ Thomas Elbert, Brigitte Rockstroh \\ Universität Konstanz, Germany
}

Johannes Krämer, Werner Lutzenberger

Universität Tübingen, Germany

Paul Grossmann ${ }^{2}$

Universität Freiburg, Germany

The present study investigated the effects of biofeedback of arterial blood pressure on cortical, peripheral, and psychological measures and the dependence of these effects on nicotine. Four groups of subjects, nonsmokers, and habitual smokers who smoked cigarettes during the experimental sessions containing $0.3,0.8$, or $1.5 \mathrm{mg}$ nicotine, respectively, participated in a feedback paradigm in which continuous feedback of mean blood pressure was provided for intervals of $8 \mathrm{~s}$ each. While tonic blood pressure did not differ between the groups, the ability to modulate blood pressure (under feedback conditions) was restricted in smokers as compared to nonsmoking subjects; increasing nicotine dosage was accompanied by poorer performance. Independently of habitual smoking and nicotine doses, heart rate increased during feedback and under conditions of blood pressure increase. In smokers, activity in the alpha band was reduced in a dose-dependent manner. Slow cortical potentials (SCPS) during the feedback interval varied with self-induced blood pressure changes

\footnotetext{
${ }^{1}$ This experiment was supported by Reemtsma Inc. Hamburg, which also provided the experimental cigarettes.

${ }^{2}$ Address correspondence to Prof. Dr. Niels Birbaumer, Department of Clinical and Physiological Psychology, Universität Tübingen, Gartenstrasse 29, 74 Tübingen, Germany.
} 
in nonsmokers (blood pressure increase was accompanied by reduced surface-negative potential shifts and vice versa), while SCP variations during feedback conditions were small in smokers, more so under the influence of 0.3 and 0.8-mg nicotine, less so under $1.5 \mathrm{mg}$. Verbal reports suggest that awareness of performance strategies may not be a necessary variable for performance on the blood pressure regulation task.

Descriptor Key Words: biofeedback; blood pressure; nicotine; slow cortical potentials.

\section{INTRODUCTION}

An increase in arterial blood pressure stimulates baroreceptor firing via afferent pathways to the Nucleus Tractus Solitarius of the brain stem, which in turn releases vagal activity and inhibits sympathetic outflow. Parallel to this efferent, homeostatic cardiovascular reflex, the baroreceptors are known to exert their influence on a number of structures ascending from the brain stem and probably also from the hypothalamus to higher cortical regions. The baroreceptors send their afferents to the insular cortex and may also influence cortical functioning via modulation of the ascending reticular formation. Available evidence suggests that an increment in baroreceptor firing constrains reticular activation, the dampening effects of which become manifest at various levels, from the spinal reflexes up to cortical excitability (Elbert \& Rau, 1991). The inhibitory effect on higher structures may explain some of the behavioral consequences of baroreceptor activation: pain tolerance increases (Dworkin, Filewich, Miller, Craigmyle, \& Pickering, 1979; Randich \& Maixner, 1984; Elbert, Birbaumer, Lutzenberger, Rockstroh, Kessler, \& Pietrowsky, 1988), anxiety is reduced, and the avoidance behavior to aversive stimuli becomes less vigorous (see Dworkin, 1988; Rau \& Elbert, 1991, for a summary of evidence). Under extreme stimulation of the baroreceptors, animals become drowsy and fall asleep (Koch, 1932). Based on such evidence, Dworkin (1988) has proposed that phasic blood pressure responses may become instrumentally reinforced if increases in blood pressure under aversive or stressful environmental conditions are followed by baroreceptor mediated cortical inhibition, ultimately resulting in reduced impact of external stimulation. Available evidence, indeed, suggests that blood pressure may be learned instrumentally (Dworkin, 1988); recently we have demonstrated a potent instrumental conditioning of phasic blood pressure in humans and have shown that conditioned pressor responses inhibit electrocortical activity (Elbert, Roberts, Lutzenberger, \& Birbaumer, 1991a). The aims of the present study were to replicate this finding and to assess the effects of cigarette smoking on the ability to modulate phasic blood pressure and its electrocortical consequences. 
A considerable body of evidence specifies nicotine as one important risk factor for the development of cardiovascular disorders, in particular coronary heart diseases. Furthermore, nicotine intake seems to interact with other risk factors such as high blood pressure, high HDL/LDL, or certain behavior patterns (Erdman, 1990). There exists a high affinity for nicotine at the nicotinergic acetylcholinergic receptors (which play a major role in pre- and postganglionic parasympathetic neurons), at postganglionic vasodilator neurons, at the resistance vessels of the muscles, and probably also in a number of central nervous system structures, not all of which have been completely identified. Nicotine exhibits a biphasic effect on acetylcholinergic receptors: the short-term agonistic effect is followed by long-term blockade of these receptors. Furthermore, nicotine stimulates the adrenal medullary hormones, adrenalin and noradrenalin, both of which act as vasoconstrictor agents and enhance heart rate and blood pressure.

While the effect of nicotine on blood pressure has been studied under various conditions such as the resting state and mental load (Ashton \& Stepney, 1982), it seems also of interest to evaluate the effect of nicotine on the modulation of blood pressure. Utilizing the same procedures of biofeedback as described in Elbert et al. (1991a), the present study addressed the impact of cigarette smoking on the capacity to modulate blood pressure. It also examined to what extent this ability would be influenced by the nicotine content of cigarettes smoked during the experiment. If nicotine blocks the cholinergic receptors in the parasympathetic pathways in a dosedependent manner, we might expect the reactivity and, hence, the short-term modulation of blood pressure to be impaired, and this effect should increase with increasing nicotine level. A reduction in vagal cardiac control in smokers was reported by Hayano, Yamada, Sakakibara, Fujinami, Yokoyama, Watanabe, and Takata (1990). Inactivation of the vagal output may cause a weakening of the baroreceptor reflex by nicotine effects and might constitute another risk factor for the development of cardiovascular disorders, because the inhibition of transient blood pressure increases as mediated by the baroreceptors might not be counteracted any more. On the other hand, such a weakening of the baroreceptor reflex could also hinder a baroreceptor mediated negative reinforcement of blood pressure increases as proposed by Dworkin. In addition, direct central nervous system effects of nicotine are assumed to constitute reinforcing, stress-reducing mechanisms if smoking counteracts decrements in concentration or performance. In order to establish nicotine effects of various levels, EEG power spectra and slow cortical potentials were measured as central nervous system measures and heart rate as an autonomic variable. In addition, the impact of conscious awareness of strategies related to 
successful blood pressure modulation was examined by verbal reports and rating scales. The multilevel recording aimed at investigating the interaction of phasic central nervous inhibition as a consequence of blood pressure increases and the contrasting effects of nicotine on (reduced) peripheral reactivity and (increased) cortical activation as indicated by reduced alpha activity and increased attentional capacity.

\section{METHOD}

\section{Subjects}

Forty male volunteers, 10 nonsmokers and 30 habitual smokers (age range 20-35 years), participated in the study. Subjects smoked 10-20 cigarettes per day with an average nicotine content of $0.8 \mathrm{mg}$ per cigarette for more than 3 years. Subjects who were under current medication or suffered from any central nervous or cardiovascular abnormality were rejected from the sample. Three subjects were excluded owing to frequent excessive ocular artifacts, contaminating their EEG recordings. Smokers were randomly assigned to one of three groups, which differed with respect to the nicotine content of the cigarettes that were smoked during the experiment: Nine subjects received cigarettes containing $0.3 \mathrm{mg}$ nicotine, ten subjects received cigarettes containing $0.8 \mathrm{mg}$ nicotine, and nine smokers smoked cigarettes with $1.5 \mathrm{mg}$ nicotine. Subjects were reinforced by earning about 2 German Marks (DM; about $\$ 1.50$ ) for each correct response, whereas they lost the same amount for each incorrect response; no subject was paid less than 30 or more than $100 \mathrm{DM}$ per session.

\section{Design and Procedure}

Heart rate and blood pressure were continuously recorded from the finger during the training to regulate blood pressure. Continuous visual feedback was provided for beat-by-beat changes in mean arterial blood pressure for trials of $8 \mathrm{~s}$ duration. The feedback stimulus was the outline of a small rocket ship that appeared on a $30 \times 40 \mathrm{~cm}$ TV screen situated $2 \mathrm{~m}$ in front of the subject at eye level. The rocket moved back and forth in a horizontal plane through a gap created by an upper and lower vertical bar, bars being situated slightly to the left of center on the TV-screen. Trials were designated by presentation of the rocket in the gap, together with the letter "A" or "B" at the right boundary of the screen. The subject's task was to move the rocket out of the gap toward the letter on each trial. Assignment of the blood pressure responses - increase and decrease - to the discriminative stimuli, the letters " $\mathrm{A}$ " or " $\mathrm{B}$," varied randomly between 
subjects. Correct responding moved the rocket toward the right and incorrect responding to the left on both types of trial. Thus, forward movements of the rocket constituted feedback of success, whereas backward movements represented error feedback to the subject. The position of the rocket remained fixed at the starting point for the first second of each feedback trial; during this time the initial change in mean arterial pressure was measured.

Systolic and diastolic arterial pressures were determined on-line for each R-R interval (maximum and minimum values, respectively, of the FinAPres Recording). The position of the rocket on each feedback trial was a linear function of the integral of mean blood pressure $(2 / 3$ diastolic plus $1 / 3$ systolic), referred to a 4 -s pretrial baseline and updated with each heart beat. If the mean change in pressure exceeded $5 \mathrm{~mm} \mathrm{Hg}$, the outline of the rocket became filled, signaling that the subject had earned a "win" point (worth $2 \mathrm{DM}$ ).

To assess control of responding in the absence of feedback, "transfer" trials were included on which the letter " $A$ " or the letter " $B$ " was presented but feedback was removed. Subjects were told that they should produce the required response as best as they could, even though feedback and win points would not be displayed to tell them how well they had done. Each session commenced with a block of 30 feedback trials (15 "A", 15 "B") followed by a block of 20 transfer trials ( 10 of each kind). This sequence was then repeated once to give a total of 100 trials for the session. Within blocks, " $\mathrm{A}$ " and " $\mathrm{B}$ " trials were given in a mixed order. The time lapsing from the end of one trial to the onset of the next pretrial baseline varied randomly between 4 and $12 \mathrm{~s}$.

Each subject participated in two identical sessions, during which heart rate, respiration, and blood pressure from the finger were recorded continuously. In addition EEG and EOG were recorded in the second session. Sessions were scheduled on consecutive days. During both sessions, smokers were requested to smoke a single cigarette prior to the experimental period and between the two blocks of 50 trials ( 30 feedback and 20 transfer trials). Subjects were asked not to smoke an hour before the experiment. This was verified during the 1-hour baseline procedure in the lab, during which subjects filled out questionnaires and electrode attachment was completed. Cigarettes (with trade names being removed) varied in their content of nicotine but were otherwise identical, specifically manufactured for experimental purposes. For nonsmokers a brief rest was scheduled between the two trial blocks.

At the end of the second session subjects were asked without prior notification to complete two questionnaires which required that subjects rate on 7 -point scales $(3=$ a great deal, $-3=$ the opposite pole, 
midpoint $=0$ ) the degree to which the following specific activities were used to achieve success on "A" and "B" trials: (1) tense muscles versus relax muscles; (2) a rapid movement versus remaining still; (3) fast, deep breathing versus slow, shallow breathing; (4) suspended versus normal breathing; (5) emotional tension versus relaxation; (6) imagining aversive versus pleasant situations; (7) concentration versus distraction; (8) observing versus not observing the rocket; (9) thinking versus not thinking about a strategy; and (10) imagining versus not imagining concrete objects. In the second questionnaire, subjects rated, in the same manner, to what extent they had paid attention to the following items: (1) the TV-screen, (2) the moving rocket ship, (3) the symbols indicating the direction of blood pressure change, (4) their own bodies, (5) movements, (6) emotional responses, (7) noises, (8) pulsatile sensations, and (9) objects in the room. An additional scale required that subjects rate their perceived success on "A" and " $B$ " trials ( $3=$ very successful; $-3=$ totally unsuccessful).

\section{Apparatus and Physiological Recordings:}

Arterial blood pressure was monitored continuously by means of an inflatable cuff (Ohmeda FinAPres) attached around the second phalanx of the left middle finger. Changes in blood volume consequent to each heart beat were sensed by a photoplethysmograph and fed to a special servo-mechanism that regulated cuff pressure to equal that of the finger's arteries (Wessling, Settles, \& de Witt, 1986; Peñaz, 1973). Because transmural pressure was maintained near zero by servo-regulation, the FinAPres transducer could be worn comfortably, although pulsatile sensations produced by the beating heart were noticeable and faded in and out as pressure in the cuff was adjusted. Hand and arm position remained constant throughout the sessions in order to avoid artifactually induced changes in blood pressure measurement. The output of the Ohmeda device was sampled at $100 \mathrm{~Hz}$ by a PDP $11 / 73$ computer. Blood pressure was also obtained from the left arm before and after each session using an automated Riva-Rocci cuff.

The electroencephalogram (EEG) was recorded along the mid-sagittal line $(\mathrm{Fpz}, \mathrm{Fz}, \mathrm{Cz}, \mathrm{Pz}$, and $\mathrm{Oz}$ ) according to the international $10-20$ system. Readings were referred to the midpoint of a $10-\mathrm{k} \Omega$ shunt attached between the earlobes. The skin at each site was cleaned with alcohol and abraded by rubbing in Omniprep abrasion paste to reduce impedance below $5 \mathrm{k} \Omega$. Nonpolarizable silver/silver-chloride electrodes (ZACK) coated with Grass EC2 paste were then affixed. A single ground electrode was attached to the neck. The EEG was amplified by a Nihon/Kohden amplifier with a time constant of ten seconds and sampled at $100 \mathrm{~Hz}$. Vertical and 
radial electrooculograms (EOGs) were derived from two electrodes (Beckman) attached $0.5 \mathrm{~cm}$ below each eye. Beckman electrode jelly served as electrolyte. The average potential of these electrodes was subtracted from the Fpz electrode to obtain the vertical EOG, and from the average earlobe potential to obtain the radial EOG (Elbert, Lutzenberger, Rockstroh, \& Birbaumer, 1986). These measures were used to screen the EEG channels for eye movement artifacts. Trials with ocular peaks of more than $80 \mu \mathrm{V}$ were rejected from the EEG-average.

The electrocardiogram (ECG) was recorded from the lower rib cage (leads V1-V5). $R$-waves were determined with a precision of $1 \mathrm{~ms}$ by the computer. Heart rate was calculated for successive 0.1 -s intervals by weighing each beat according to the proportion of the interval it occupied.

Respiration was measured from a pneumatic belt placed around the lower chest and sampled at $100 \mathrm{~Hz}$.

\section{Analysis of Physiological Data}

Blood pressure and heart rate were analyzed for both sessions, the EEG was additionally analyzed for the second session. Trials containing excessive vertical or radial eye movements $(>70 \mu \mathrm{V})$ or extraneous EEG shifts exceeding $70 \mu \mathrm{V}$ in any channel or other obvious artifacts were rejected from the analysis. Four subjects, having more than one third of artifact-contaminated trials were excluded from the sample. Eighteen percent of the trials were rejected for one or more of these reasons. After artifact rejection, data of 36 subjects (eight nonsmokers, nine $0.3-\mathrm{mg}$ nicotine smokers, ten $0.8-\mathrm{mg}$ nicotine smokers, and nine $1.5 \mathrm{-mg}$ nicotine smokers) were available for further analysis. Continuous physiological measures (systolic, diastolic, and mean blood pressure, heart rate, respiration depth and frequency, EEG and EOG) were collapsed to $100-\mathrm{ms}$ intervals by a phase-free digital filter. The slow potential data were transformed into a $\mathrm{DC}$-record applying the transformation formula by Elbert \& Rockstroh (1980). Change scores were then computed for each measure by subtracting the mean for the last seven seconds of the trial from the mean observed during the last second of the pretrial period. Furthermore, two overlapping 2.56-s epochs starting $1 \mathrm{~s}$ after trial onset were cut out of each original EEG-trace and submitted to fast Fourier transformations. The two resulting power spectra were first averaged for each trial and then across artifact-free trials. A measure for EEG-alpha activity was obtained as the average power in the range from 8 to $12 \mathrm{~Hz}$. Respiration data were collapsed to $100-\mathrm{ms}$ values. Two approaches were used to analyze respiratory changes: First, data were averaged in the same way as the 
cardiovascular recordings to evaluate systematic patterns, second, mean power spectra were calculated based on 12.8-s epochs of each trial.

Scores for each physiological response were subsequently averaged over blood-pressure increase and decrease trials separately, in each feedback and transfer block. This yielded 8 summary measures per subject in each session for each physiological variable. Summary measures were then averaged across subjects for each group. Unless otherwise indicated, these data provided the basis for statistical tests (ANOVAs and Student's $t$ ) with the between-subject factor GROUPS (comparing nonsmokers with the three groups of smokers in one ANOVA and comparing the three groups of smokers in another ANOVA) and the within-subject factors Differentiation (of the respective measure on required blood pressure increase versus required blood pressure decrease). For the statistical analysis of electrocortical responses the ANOVA included an additional within-subject factor Topography, comparing responses at the different recording sites (Fpz-Fz-Cz-Pz-Oz).

\section{Analysis of the Verbal Reports}

The ratings that subjects gave on the scales assessing specific response strategies were analyzed by computing bidirectional differences (for " $\mathrm{A}$ " minus "B") for each subject on each scale. It was then determined whether the bidirectional scores for each scale differed significantly from zero $(t-$ tests). In addition, correlational analyses (Pearson) served to confirm a relationship between subjective ratings of executed strategies and performance on the feedback task.

\section{RESULTS}

\section{The Effects of Cigarette Smoking on Blood Pressure Levels}

Mean blood pressure values were compared between groups in order to establish potential baseline differences that could have influenced the acquisition of blood pressure control. Averaged across all Riva-Rocci-measurements (prior to and following the first and the second session) the mean group values differed by less than $4 \mathrm{~mm} \mathrm{Hg}$. Neither the mean arterial blood pressure nor the mean systolic and the mean diastolic blood pressure differed significantly between the groups of smokers. It is interesting to note that the mean blood pressure values in habitual smokers who were smoking during the experiment $(99.4 \mathrm{~mm} \mathrm{Hg})$ were not elevated compared to nonsmokers $(102 \mathrm{~mm} \mathrm{Hg}$ ) (FinAPres values are usually higher than Riva-Rocci levels; Peñaz, 1973). 


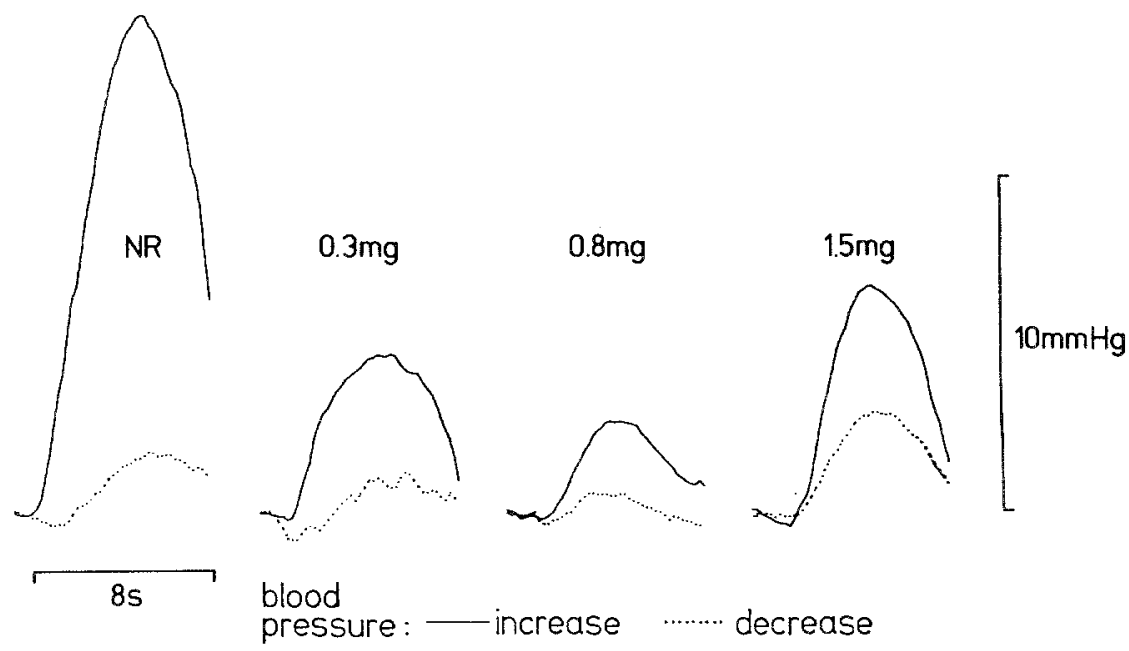

Fig. 1. Blood pressure responses (in $\mathrm{mm} \mathrm{Hg}$ ) during the feedback condition, averaged separately for trials with required blood pressure increase (solid) and decrease (dotted) trials and averaged separately for the four groups.

\section{The Effects of Cigarette Smoking on Blood Pressure Response}

Subjects within all groups achieved control over their phasic blood pressure in that they produced systematic differences depending on the discriminative stimuli for blood pressure increase and blood pressure reduction. Figure 1 illustrates the average conditioned response for trials with required blood pressure increase and trials in which a blood pressure reduction was rewarded. While blood pressure was easily enhanced above pretrial baseline, reductions were not consistently achieved. Averaged across all subjects, a mean arterial blood pressure of $117 \mathrm{~mm} \mathrm{Hg}$ was achieved under conditions of blood pressure increase, while a mean of $113.6 \mathrm{~mm} \mathrm{Hg}$ was obtained under conditions of blood pressure reduction. Significant main effects of Differentiation confirmed that responses were differentially conditioned: mean arterial pressure: $F(1,32)=17.6, p<.01$; systolic: $F(1,32)=11.6, p<.01$, diastolic: $F(1,32)=19.0, p<.01$. Although the mean blood pressure was higher during feedback (116.8 $\mathrm{mm} \mathrm{Hg}$ ) than during transfer trials $(114.6 \mathrm{~mm} \mathrm{Hg}, F(1,32)=7.3, p<.05)$, differentiation was larger under transfer than under feedback conditions (Differentiation $\times$ Condition interaction: $F(1,32)=9.3, p<.01$ ). 
Table I. Mean values and standard deviations for the differences between required increase and decrease of blood pressure.

\begin{tabular}{lllll}
\hline & $\begin{array}{c}\Delta \text { Blood } \\
\text { pressure } \\
(\mathrm{mm} \mathrm{Hg})\end{array}$ & $\begin{array}{c}\Delta \text { Heart rate } \\
(\mathrm{bpm})\end{array}$ & $\Delta \mathrm{Fz}(\mu \mathrm{V})$ & $\begin{array}{c}\Delta \text { Respiration } \\
\text { power } \\
(\%)\end{array}$ \\
\hline $0.3 \mathrm{mg}$ & $3.2(1.8)$ & $4.6(3.0)$ & $-2.1(4.5)$ & $1.1(4.1)$ \\
$0.8 \mathrm{mg}$ & $1.8(0.7)$ & $2.2(1.8)$ & $3.3(2.2)$ & $1.8(1.5)$ \\
$1.5 \mathrm{mg}$ & $2.8(2.0)$ & $1.6(1.2)$ & $3.7(3.9)$ & $3.0(1.0)^{*}$ \\
$\mathrm{NS}$ & $10.6(2.7)^{* *}$ & $5.0(2.9)$ & $18.0(7.6)^{*}$ & $2.0(1.0)$ \\
$F$ differentiation & $17.6^{* *}$ & $8.6^{* *}$ & $5.8^{*}$ & $<1$ \\
$F$ group $\times$ diff & $4.5^{*}$ & $<1$ & 2.82 & $<1$ \\
\hline
\end{tabular}

${ }^{*} p<0.05,{ }^{* *} p<0.01$.

Feedback success was most pronounced in nonsmokers with a difference of $10.6+2.7 \mathrm{~mm} \mathrm{Hg}$ in the blood pressure responses averaged across the last $7 \mathrm{~s}$ of the feedback interval $(t(7)=3.6, p<.01)$. Table I shows a summary of the differences of the responses. The ability of smokers to modulate blood pressure was reduced and not consistently significant: for the group smoking cigarettes with $0.3 \mathrm{mg}$ nicotine the mean differentiation amounted to $3.2 \pm 1.8 \mathrm{~mm} \mathrm{Hg}$ (n.s.); smoking $0.8 \mathrm{mg}$ cigarettes allowed only $1.8 \pm 0.7 \mathrm{~mm} \mathrm{Hg}(t(9)=2.5, p<.05)$, and smoking $1.5 \mathrm{mg}$-cigarettes resulted in $2.8 \pm 2.0 \mathrm{~mm} \mathrm{Hg}$ differentiation (n.s.). These differences are illustrated in Figure 1 and were confirmed by Group $\times$ Differentiation interaction effects for mean arterial blood pressure $(F(3,32)=4.5, p<.05)$, systolic $(F(3,32)=3.8, p<.05)$ and diastolic arterial pressure $(F(3,32)=$ $4.2, p<.05)$. The nonsmokers' bidirectional control of phasic blood pressure turned out to be more pronounced than that of the smoker groups: non smokers vs. $0.3 \mathrm{mg}: t(15)=2.0, p<.05$; vs. $0.8 \mathrm{mg}: t(16)=3.2$, $p<.01$; vs. $1.5 \mathrm{mg}: t(15)=2.2, p<.05$ ).

The acquisition of blood pressure control was also examined by comparing the net amount of win points between the two sessions. Only nonsmokers could increase the amount of win points significantly from 13 to $35(t(8)=2.2, p<.01$ ), while no significant changes were found in the three groups of smokers. (For the groups of smokers and the two sessions net win scores were respectively 13 and 10 for smokers of cigarettes with $0.3 \mathrm{mg}$ nicotine, 7 and 9 for $0.8 \mathrm{mg}$ nicotine, and 11 and 11 for $1.5 \mathrm{mg}$ nicotine). This result is confirmed by an interaction Groups $\times$ Differentiation with $F(3,32)=3.35(p<.05)$. Obviously only nonsmokers learned to improve blood pressure control in the second session. The amount of nicotine consumed with each cigarette does not seem to affect a performance increment. 

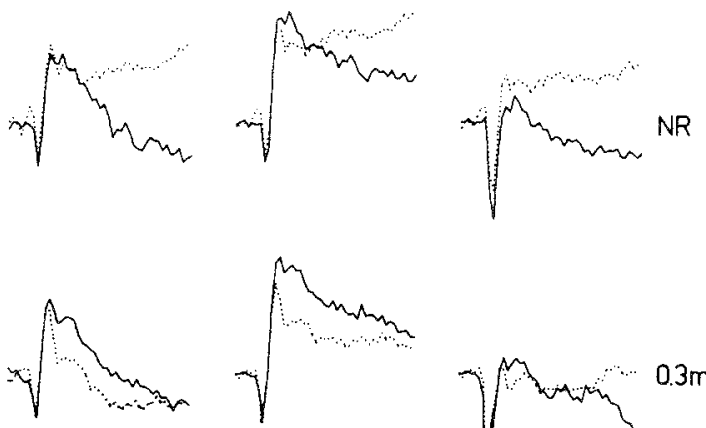

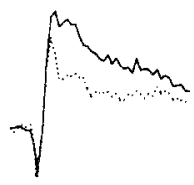

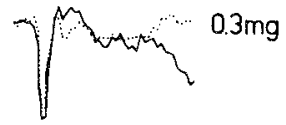

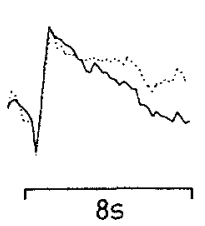

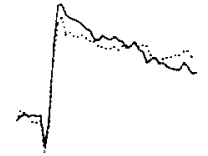

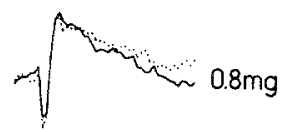

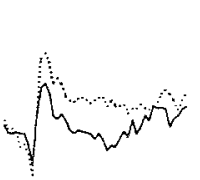

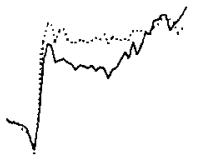<smiles>C[14CH2][14CH2][14CH2]</smiles>

$F_{Z}$

$\mathrm{C}_{\mathrm{Z}}$

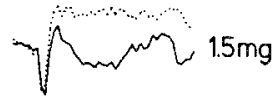

$P_{z}$

blood

pressure : - increase ….... decrease

Fig. 2. Slow cortical potentials (in muV) during the $8 \mathrm{sec}$ feedback interval averaged separately for trials with required blood pressure increase (solid) and blood pressure decrease (dotted lines), the four groups, and three EEG recordings along the mid-saggital line (Fz: frontal, $\mathrm{Cz}$ : central, $\mathrm{Pz}$ : parietal) referred to earlobes reference. Negativity is up.

\section{Effects of Blood Pressure Modulation on Autonomic Variables}

Averaged across all subjects, mean heart rate increased to $81 \mathrm{bpm}$ under conditions of blood pressure increase, while a mean of $77.8 \mathrm{bpm}$ was measured under conditions of blood pressure reduction (Differentiation: $F(1,32)=8.6, p<.01)$. Higher values $(80 \mathrm{bpm})$ were measured during feedback than during transfer trials $(77.4 \mathrm{bpm}$, Condition: 
$F(1,32)=41.9, p<.01)$. In smokers, the mean heart rate increased by 5.8 $\mathrm{bpm}$ on the average under conditions of blood pressure increase and decreased by $0.4 \mathrm{bpm}$ under conditions of blood pressure reduction $(F(1,26)=5.1, p<.05)$. Nicotine dosage did not significantly affect heart rate modulation.

No significant patterns were obtained for the averaged respiration $(F<1)$. The analysis of the total power of the respiration showed nonsignificant effects of Differentiation $(F(1,32)=2.8)$ and of Group $\times$ Differentiation $(F<1)$ but a significant effect of Groups $(F(3,32)=6.8$, $p<.001)$ : Nonsmokers showed significantly more power than each of the smoking groups while no significant differences between the smokers were found.

\section{Effects of Blood Pressure Modulation on Electrocortical Measures}

In nonsmokers a slow negative potential shift, comparable to a contingent negative variation, developed during the 8-s blood pressure regulation interval, whenever blood pressure decrease was required and achieved. Negativity was markedly reduced during or consequent upon blood pressure increase (see Fig. 2). Considering the last $7 \mathrm{~s}$ (mean change from baseline without the evoked potential), the difference in slow potentials between the conditions amounted to $5 \mu \mathrm{V}$ (significant main effect of Differentiation at two recording sites: for $\mathrm{Fz} t(7)=2.36, p<.05$ and for $\mathrm{Cz} t=2.04, p<.1$ ).

On the other hand, Figure 2 illustrates that the slow potential differentiations parallel those for the blood pressure responses in smokers, primarily in subjects smoking $0.3-\mathrm{mg}$ and $0.8-\mathrm{mg}$ nicotine cigarettes during the experiment. Only SCPs of subjects smoking cigarettes of $1.5 \mathrm{mg}$ nicotine resemble those of nonsmokers. These differences between groups were confirmed by a Group $\times$ Differentiation interaction with $F(3,32)=2.82$, $p<.05$.

Smoking of cigarettes with medium $(0.8 \mathrm{mg})$ and high $(1.5 \mathrm{mg})$ nicotine content reduced the activity in the alpha band $(8-12 \mathrm{~Hz})$. A parietal maximum with $5.0 \mu \mathrm{V}^{2}$ was found, followed by $4.4 \mu \mathrm{V}^{2}$ at $\mathrm{Cz}$ and $\mathrm{Fz}$ and $4.1 \mu \mathrm{V}^{2}$ at $\mathrm{Oz}$ (main effect of Topography with $F(3,96)=9.1, p<.01$ ). For the parietal recording, alpha activity reached $4.8 \mu \mathrm{V}^{2}$ in nonsmokers and $5.3 \mu \mathrm{V}^{2}$ in smokers smoking $0.3 \mathrm{mg}$ nicotine cigarettes, but was reduced under the influence of $0.8 \mathrm{mg}$ nicotine $\left(3.6 \mu \mathrm{V}^{2}\right)$ and $1.5 \mathrm{mg}$ nicotine (4.0 $\left.\mu \mathrm{V}^{2}\right)$. The group differences are confirmed by a main effect Groups with $F(3,32)=3.8(p<.05)$. In particular subjects smoking $0.8 \mathrm{mg}$ nicotine cigarettes exhibited significantly lower activity in the alpha range than nonsmokers $(t(16)=2.4, p<.05)$ and $0.3 \mathrm{mg}$ nicotine cigarette smokers 
$(t(17)=2.3, p<.05)$. These results were further confirmed by regression analysis: lower nicotine content of the smoked cigarette covaried with the higher activity in the alpha range $(r=-.32, F(1,34)=4.0, p<.05)$.

\section{Verbal Reports}

On the seven-point rating scale assessing for the subjective estimate of success and failure on the task of blood pressure modulation, smokers evaluated themselves as not particularly successful (mean .28 under conditions of blood pressure increase), while their success in decreasing the blood pressure was rated as slightly unsuccessful $(-1.2$; for the difference $t(26)=2.97, p<.01$ ). Nonsmokers, on the other hand, realized their success on blood pressure increase with 1.9 , compared to -1.3 for the blood pressure decrease $(t(8)=12.3, p<.01)$. A correlational analysis (Pearson's $r$ ) of performance data and subjective ratings indicates that performance is not necessarily perceived consciously (i.e., so that it is verbalized): For nonsmokers - who exhibited superior performance the relationship was positive but nonsignificant with $r=.43(p=.16)$ for both conditions, blood pressure decrease as well as blood pressure increase. On the other hand, significant correlation coefficients were found for the three smoking groups, who obviously rated their (poorer) performance adequately for the condition of blood pressure increase ( $r=.74, p<.05$ for subjects smoking $0.3 \mathrm{mg}$ cigarettes, $r=.72, p<.05$ for subjects smoking $0.8 \mathrm{mg}$ cigarettes, $r=.67, p<.05$ for subjects smoking $1.5 \mathrm{mg}$ nicotine cigarettes). Regarding conditions of blood pressure decrease, none of the correlations reached significance, indicating that performance under this condition is independent of the subjective evaluation of performance.

In order to determine by which strategies subjects achieved control of their blood pressure during feedback, ratings on ten seven-point scales of executed activities were analyzed. Nonsmokers showed a significant differentiation on the scale evaluating perceived muscle tension: muscle tension was increased on blood pressure increase and reduced on blood pressure reduction trials (difference score $3.0, t(9)=2.75, p<.05$ ). Furthermore, nonsmokers increased their state of readiness when blood pressure increase was required, while lowering it when blood pressure decrease was required (mean difference $4.7, t(9)=22.1, p<.01$ ). When items were clustered into "central / mental activities" versus "peripheral activities", central activities differed to a larger extent between conditions of blood pressure modulation $(7.8, t(9)=3.6, p<.05)$ than peripheral activities $(5.4, p<.1)$. On the other hand, imagining scenes was the only scale differentiating between conditions of blood pressure modulation in 
smokers $(t(27)=2.1, p<.05)$. (This effect was mainly due to a strong differentiation on this scale in the group smoking $0.3 \mathrm{mg}$ cigarettes (2.3, $t(8)=2.5, p<.05)$. While ratings did not correlate significantly with performance values (scores) in nonsmokers, applying "peripheral activities" to modulate blood pressure was positively related to blood pressure increase in nonsmokers $(r=.42, p<.05)$ but negatively related to blood pressure decrease $(r=-.54, p<.01)$. On the other hand, smokers tended to execute "central activities" when blood pressure increase was required $(r=+.47)$ but generally decreased such activities on the attempt to lower blood pressure $(r=-.41, p<.05)$.

\section{DISCUSSION}

In the present sample, nicotine does not affect tonic blood pressure in young subjects, but it obviously inhibits self-induced modulation of blood pressure. Specifically, it lowers the ability to raise the blood pressure within seconds upon command. As there were no restrictions as to how such a blood pressure response might have been achieved, we can assume that this inability would also extend to conditions in everyday life, not only in situations where a voluntary modulation of blood pressure is required, which is of course not the usual case. Thus, on the one hand, cigarette smoking seems to restrict the organism's capacity for blood pressure adaptation; on the other hand, it might make subjects less prone to elevated levels of blood pressure, in particular when procedures of instrumental conditioning play a role. Dworkin (1988) has argued that operant procedures may play a role for the development of hypertension. Stressful conditions might be perceived as less aversive, when the subject raises his blood pressure, as the subsequently elevated firing of baroreceptors reduces cortical arousal.

The present results obtained from nonsmokers confirm that an enhancement of blood pressure lowers cortical excitability, as slow surface negativities can be considered to indicate such changes (Rockstroh et al., 1989; Birbaumer et al., 1990; Elbert, 1991). In a number of studies, we have previously shown that stimulation of arterial pressoreceptors reduces cortical negativity and dampens CNS excitability from the cortex (Elbert et al., 1988; Elbert, Tafil-Klawe, Rau, \& Lutzenberger, 1991b) down to the spinal reflexes (Elbert \& Rau, 1991). The present results indicate that such a relationship also holds for the different groups: The less pronounced the blood pressure differentiation, the smaller the differences in slow potentials. We have previously observed this relationship for nonsmokers (Elbert, et al. 1991a). 
The effects of smoking confirm the overall picture of nicotine causing phasic inhibitory and excitatory physiological patterns at different levels of the organism. (However, it should be noted that the present sample comprised only male subjects. Previous studies from our laboratory have shown female subjects to respond in a different manner to smoking cigarettes of various nicotine content.) Excitatory effects were indicated by reduced activity in the alpha band. Smoking produced an increased thalamocortical excitation in a dose-dependent manner: with increased nicotine content of the smoked cigarette, alpha activity decreased, indicating decreased thalamo-cortical inhibition. Simultaneous inhibition of cortical excitability mediated by baroreceptor firing - was neutralized by cigarette smoking. The significant relationships between performance on the blood pressure modulation task and verbalized strategies may indicate enhanced selective attention in smokers to central and peripheral processes during task performance. This may increase the smoker's sensitivity to bodily processes ("visceral perception").

In previous studies we have shown that the ability to modulate slow cortical potentials was enhanced after smoking cigarettes of high nicotine content (Birbaumer \& Schmidt, 1990), indicating a gain in cortical "flexibility of attention." The present study demonstrated impaired ability of self-regulation of an autonomic variable under the influence of nicotine. It is tempting to relate this effect to the anticholinergic influence of nicotine on the parasympathetic nervous system. This would constitute the prerequisite condition for the peripheral arc of the baroreceptor reflex, rendering subjects unable to modulate their cardiovascular system (see also Hayano, Yamada, Sakakibara, Fujinami, Yokoyama, Watanabe, \& Takata, 1990). Although it remains to be examined whether the impaired capability of blood pressure modulation is due to the actual nicotine intake or to the habitual smoking, we may assume that the tonic effects of habitual smoking should not differ fundamentally from the phasic effects, as the effects of nicotine on peripheral and central nervous systems are the same as long as nicotine is circulating in the blood (about $20 \mathrm{~min}$ after smoking).

It may critically be argued that the differences between smokers and nonsmokers may be due to another behavioral, psychological, or physiological factor unrelated to the smoking habit and the nicotine. Some facts speak against such an interpretation; however, such a third factor cannot be excluded entirely: The nicotine dose-dependent effects on EEG alpha power may indicate a direct relationship with the independent variable. Previous studies from our group (Elbert \& Birbaumer, 1987) did also show clear dose dependence of cortical and autonomic variables. 


\section{REFERENCES}

Ashton, H., \& Stepney, R. (1982). Smoking, psychology and pharmacology. London: Tavistock. Birbaumer, N., \& Schmidt, R. F. (1990). Biologische Psychologie (pp. 587-588). Heidelberg: Springer-Verlag.

Birbaumer, N., Elbert, T., Canavan, A., \& Rockstroh, B. (1990). Slow potentials of the cerebral cortex and behavior. Physiological Reviews, 70, 1-41.

Bonvallet, M., Dell, P., \& Hiebel, G. (1953). Sinus carotidien et active electrique cerebrale. Comptes Rendus des Seances de la Societe de Biologie et de Ses Filiales (Paris) 147, 1166-1169.

Dworkin, B. R. (1988). Hypertension as a learned response: The baroreceptor reinforcement. In T. Elbert, W. Langosch, A. Steptoe, \& D. Vaitl, (Eds.) Behavioral medicine in cardiovascular disorders. Chichester: John Wiley.

Dworkin, B. R., Filewich, R. J., Miller, N. E., Craigmyle, N., \& Pickering, T. G. (1979). Baroreceptor activation reduces reactivity to noxious stimulation: Implications for hypertension. Science, 205, 1299-1301.

Elbert, T. (1991). Slow cortical potentials reflect the regulation of cortical excitability. In McCallum (Ed.). Slow potential changes in the human brain. New York, Plenum Press.

Elbert, T., \& Birbaumer, N. (1987). Hemispheric differences in relation to smoking. In A. Glass, (Ed.). Individual Differences in Hemispheric Specialisation. (pp. 195-206). Plenum Press: New York.

Elbert, T., Birbaumer, N., Lutzenberger, W., Rockstroh, B., Kessler, M., \& Pietrowsky, R. (1988). Baroreceptor stimulation increases pain sensation in borderline hypertensives. Psychophysiology, 25, 25-29.

Elbert, T., \& Rau, H. (1991). What goes up from heart to brain must calm down from brain to heart. Studies on the interactions between the cardiovascular and the central-nervous system. In D. Vaitl, \& R. Schandry (Eds.), Interoception of Cardiovascular Processes. Berlin: Springer-Verlag.

Elbert, T., Roberts, L., Lutzenberger, W., \& Birbaumer, N. (1991a). Modulation of slow cortical potentials by instrumentally learned blood pressure responses. Psychophysiology (in press).

Elbert, T., \& Rockstroh, B. (1980). Some remarks on the development of a standardized time constant. Psychophysiology, 17, 504-505.

Elbert, T., Tafil-Klawe, M., Rau, H., \& Lutzenberger, W. (1991b). Cerebral and cardiac responses to unilateral stimulation of carotid sinus baroreceptors. Journal of Psychophysiology (in press).

Erdman, R. A. M. (1990). Myocardial infarction and cardiac rehabilitation. In A. Kaptain (Ed.), Behavioral Medicine (pp. 127-146). Chichester Wiley, 1990.

Hayano, J., Yamada, M., Sakakibara, Y., Fujinami, T., Yokoyama, K., Watanabe, Y., \& Takata, K. (1990). Short- and long-term effects of cigarette smoking on heart rate variability. American Journal of Cardiology, 65(1), 84-88.

Koch, E. B. (1932). Die Irradiation der pressorezeptorischen Kreislaufreflexe. Klinische Wochenschrift, 2, 225-227.

Peñaz, J. (1973). Photoelectric measurement of blood pressure, volume and flow in the finger. In R. Albert, W. Vogt, \& W. Hlebig (Eds.), Digest 10th conference Med. Biol. Engineering, p. 104.

Randich, A., \& Maixner, W. (1984). Interactions between cardiovascular and pain regulatory systems. Neuroscience \& Biobehavioral Reviews, 8, 342-367.

Rau, H., Elbert, T. (1991). The role of baroreceptors in the modulation of pain perception and PRES, a controlled technique for the stimulation of baroreceptors. In D. Vaitl, \& R. Schandry (Eds.), Interoception of Cardiovascular Processes. Berlin: Springer-Verlag.

Rau, H., Elbert, T., Lutzenberger, W., Eves, F., Rockstroh, B., \& Birbaumer, N. (1988). Pavlovian conditioning of peripheral and central components of the baroreceptor reflex. Journal of Psychophysiology, 2, 119-127.

Rockstroh, B., Elbert, T., Canavan, A., Lutzenberger, W., \& Birbaumer, N. (1989). Slow brain potentials and behavior II. 2nd edition. Baltimore: Urban \& Schwarzenberg. 
Wessling, K. H., Settels, J. J., de Wit, B. (1986). The measurement of continuous finger arterial pressure noninvasively in stationary subjects. In T. H. Schmidt, T. M. Dembroski, \& G. Blümchen, (Eds.), Biological and Psychological Factors in Cardiovascular Disease (pp. 355-375). Berlin: Springer-Verlag. 